in clinical practice which are currently not covered by the European guidelines and these need to be reviewed to provide physicians with appropriate guidance.

\section{P127 SPECIAL INTEREST CLINIC: A NOVEL GENITOURINARY MEDICINE SERVICE INITIATIVE PROVIDING CONTINUITY OF CARE AND EDUCATIONAL OPPORTUNITIES}

Vian Shafiq*, Jonathan Shaw, Ashish Sukthankar. Manchester Centre for Sexual Health, Manchester, UK

\subsection{6/sextrans-2015-052126.170}

Introduction External referral to dermatology and psychosexual services from genitourinary medicine (GUM) can cause delays in patient care. To counter this within our service an experienced consultant has established a Special Interest Clinic (SIC) reviewing dermatology, erectile dysfunction and complex GUM cases. Written educational feedback is offered to internal referrers. We reviewed the impact of SIC.

Aims To evaluate the service offered by SIC.

Methods Data was collated from randomly selected patient records who attended SIC between April 2012 and April 2013.

Results A total of 100 records were reviewed. 67 patients were male, 25 of whom were MSM. Patients were ethnically diverse, White British (52) being the most common ethnicity. Median age was 33 years (range 19-70). 12 patients were HIV-infected. Internal referrals predominated (96) and average waiting time from referral was 6.2 weeks (range $0.14-28$ ). Broadly stratifying referrals 40 patients were complex GUM, 35 psychosexual medicine, 25 dermatology. The most prevalent diagnoses were erectile dysfunction (23) and lichen sclerosus (9). 9 patients required skin biopsy, 8 of which were performed within SIC. Ongoing follow up was recommended to 60 patients, of which $43(71.7 \%)$ were retained. 27 patients were discharged after first attendance. 77 referrers requested feedback, all received it.

Conclusion Keeping patients within our service provided continuity of care. The availability of formal feedback increases educational opportunities for referrers. We recommend experienced clinicians consider establishing similar SICs in other services. A challenge services will encounter is the lack of specific SHHAPT coding for prevalent SIC diagnoses.

\section{P128 MEETING STANDARDS IN MANAGEMENT OF SEXUAL ASSAULT: ARE WE THERE YET?}

Sujeevani Munasinghe, Malaki Ramogi*, Noellette L'Esperance. Colchester Hospital University Foundation Trust, Essex, UK

\subsection{6/sextrans-2015-052126.171}

Background/introduction Effective management of victims of sexual assault is important to encourage people to report abuse and receive care. BASHH provide guidance on management of sexually assaulted patients. We reviewed case notes of 36 patients who were treated for sexual assault.

Aim(s)/objectives

- To identify demographic characteristics of sexually assaulted victims attending the clinic.

- To understand how we meet the BASHH guidance (2011) in the management of sexual assaults.

- To assess the usefulness of locally used template for cases of sexual assault
Methods Case notes of 36 patients treated for sexual assault who attended the clinic from January 2013 to March 2014 were reviewed. A questionnaire was designed to collect data and the data was analysed using Microsoft excel.

Results 44 case notes were identified but 36 cases fulfilled the inclusion criteria. Of the 14 auditable outcomes, only documentation of essential criteria (standard 1) reached the 100\% standard and six achieved above $75 \%$ of the expected standard of $100 \%$. These include documentation of physical injuries, selfharm risk assessment, offer of emergency contraception, offer of active vaccination against Hepatitis B and assessment of child protection need. Offer of baseline STI screening was documented in $72 \%$. Poor documentation of BASHH criteria on further referral for physical injuries (33\%) and repeat testing for STIs (36\%) were identified.

Discussion/conclusion Importance of complete documentation on sexual assault cases should be emphasised. Reviewing the sexual assault template to capture all necessary information was identified as a result of this audit.

\section{P129 SHARED CLINICAL PRIORITIES IN AN INTEGRATED SEXUAL HEALTH SERVICE}

Gordon Scott*. Chalmers Sexual Health Centre, Edinburgh, UK

\subsection{6/sextrans-2015-052126.172}

Background/introduction Demand exceeds capacity in many sexual health services. In response to this, our GUM department developed a triage policy based on agreed clinical priorities. When we integrated with the local Sexual and Reproductive Health (SRH) service, which had its own more loosely defined priorities, an essential part of the process was to agree shared clinical priorities.

Aim(s)/objectives To create a single, agreed set of priorities across an integrated sexual health service.

Methods We reviewed the existing GUM priorities, and agreed they were still applicable. We created a formal set of SRH priorities. We merged the two into an integrated set of clinical priorities that would apply across the whole service.

Results The existing GUM priorities were patients with or at significant risk of HIV, followed by patients with or at significant risk of syphilis, then gonorrhoea, then chlamydia. The SRH priorities were widespread provision of long-acting reversible contraception (LARC), followed by emergency contraception (especially IUD), high quality abortion service, services for young people and services in more deprived areas. The single, agreed set of priorities for the integrated service were HIV-positive patients, women with unplanned pregnancy and under 16's; followed by patients at high risk of HIV, high risk of unplanned pregnancy, and/or people living in areas of high deprivation.

Discussion/conclusion Creating shared priorities has proved invaluable when pressure on the service builds up. Both services had to shed priorities that might have hitherto been regarded as "sacrosanct".

\section{P130 DOES USE OF A PRO FORMA IMPROVE MANAGEMENT OF COMPLAINANTS OF SEXUAL ASSAULT?}

Rachel Caswell ${ }^{*}$, Christine Hardwick, Penny Goold. Whittall Street Clinic, Birmingham, UK

10.1136/sextrans-2015-052126.173 
Background/introduction A large GUM clinic introduced a sexual assault pro forma to improve the management of patients alleging sexual assault.

Aim(s)/objectives To compare standard of care of complainants of sexual assault with and without use of pro forma.

Methods A retrospective review of patient records with evidence of first disclosure of sexual assault was undertaken for an eight month period. Data on 16 outcomes including 14 nationally auditable standards was analysed against use of the pro forma. Data analysis was performed using Stata. Data collection will be extended to twelve months.

Results 65 patients were included. A pro forma was only completed in 58\%. The following outcomes were significantly associated with pro forma use: HIV risk assessment $(p=<0.001)$, detailed history of assault ( $p=<0.001$ ), offer of hepatitis B vaccine $(p=0.03)$ and completion of self-harm assessment $(p=<0.001)$. Other outcomes supporting pro forma use were risk assessment of vulnerability $(p=<0.001)$ and offer of psychological support $(p=<0.001)$. STI testing specifically for hepatitis $\mathrm{C}$ and trichomonas vaginalis was below the national auditable standard in both groups.

Discussion/conclusion The use of a pro forma has improved clinical care of complainants of sexual assault. Poor uptake of use of the pro forma within the clinic needs to be addressed. Amendments to the pro forma may improve outcomes such as increasing offer of testing for hepatitis $\mathrm{C}$ and trichomonas vaginalis.

\section{P131 MANAGEMENT OF SEXUAL ASSAULT IN A COUNTY- WIDE INTEGRATED SEXUAL HEALTH SERVICE: INFREQUENTLY REPORTED BUT COMPLEX NEEDS IDENTIFIED}

${ }^{1}$ Nicola Wallis, ${ }^{2}$ Sue Ralph, ${ }^{2}$ Alison Wardropper, ${ }^{2}$ Conrad White, ${ }^{2}$ Sarah Duncan ${ }^{1}$ University of Newcastle Medical School, Newcastle, UK; ${ }^{2}$ County Durham and Darlington NHS Foundation Trust Sexual Health Service, County Durham and Darlington, UK

\subsection{6/sextrans-2015-052126.174}

Background/introduction County Durham and Darlington NHS Foundation Trust (CDDFT) recently published local standards of care for patients disclosing sexual assault within the sexual health service. CDDFT is the sole provider of sexual health services throughout the county, offering fully integrated GUM, Family Planning and HIV clinics.

Aim(s)/objectives This study aims describe the current management of sexual assault within CDDFT Sexual Health Services.

Methods Patients who disclosed an alleged sexual assault from 01.01.2014-31.12.2014 were identified by local electronic codes and retrospective case note review was performed. Summary statistics were calculated using STATA v 11.0 and means/percentages presented as appropriate.

Results Of 55 patients reporting alleged sexual assault, 44 case notes were available for review (80\%). 39 patients were female $(89 \%)$ and 5 were male $(11 \%)$ with a similar mean age of 24.5 years. Police involvement was equally prevalent in patients reporting recent verses historical sexual assault (18/28 (64\%) v $9 / 16(56 \%) \mathrm{p}=0.52)$ and incident details were recorded in all cases where police referral was declined (17/17, 100\%). 39/44 patients accepted STI screening, 7/44 patients were at risk of unwanted pregnancy and accepted emergency contraception;
12/44 patients were offered prophylactic antibiotics and 24/44 were offered Hep B vaccination; 6/44 patients commenced PEP and 6/8 under 18 year olds were referred to local safeguarding teams.

Discussion/conclusion Patients reporting alleged sexual assault were seen infrequently but often had complex needs. Implementing new local policy with strengthen our ability to identify these patients and standardise our approach to management.

\section{P132 "WHAT DO I DO WITH MY VIBRATOR DOCTOR?"}

Daisy Ogbonmwan*, Stephen Bushby. Department of GUM, Sunderland Royal Hospital, Sunderland, UK

\subsection{6/sextrans-2015-052126.175}

Background/introduction The use of sex toys is common amongst same sex relations and heterosexuals. Evidence exists that sex toys can be a method of transmission for many sexually transmitted infections (STI). Literature from BASHH and FSRH are explicit in recognising this and it is therefore important that we discuss the importance of safe sex toy use with our patients. Aim(s)/objectives Our aims were to assess whether clinicians routinely discussed the use of sex toys; if so with whom and whether advice was routinely given post diagnosis of an STI.

Methods A survey was designed and sent to local sexual health clinics. Responses were anonymous, using a web site link. Respondents were asked, amongst other questions, if they routinely discussed sex toy usage with their patients and if so when and with whom; and what advice was given regarding sex toy usage post diagnosis of an STI.

Results Responses were received mostly from consultants and specialist nurses. 25\% regularly discussed sex toys largely during safe sex discussions. Respondents felt women who have sex with women and men who have sex with men (94\%, 84\% respectively) benefitted most from this discussion. $22 \%$ discussed possible STI transmission with sex toys; however $88 \%$ did not routinely give advice post STI diagnosis.

Discussion/conclusion Results show that despite evidence there is limited discussion regarding safe sex with sex toys in this group of clinicians. This could lead to unawareness amongst our patient population and unnecessary transmission of infection. To improve this, education is to be undertaken via our regional BASHH meeting.

\section{P133 THE EFFECT OF INTRODUCING ROUTINE SELF-TAKEN EXTRA-GENITAL SWABS IN A GUM CLINIC COHORT}

Farai Nyatsanza*, Anisha Trivedy, Gary Brook. Central Middlesex Hospital, London, UK

\subsection{6/sextrans-2015-052126.176}

Background Starting July 2013, self-taken extra-genital swabs were offered routinely to all patients attending the department. Aim To assess detection of extra-genital infection since introduction of self-taken swabs.

Methods We compared patients diagnosed with Chlamydia and Gonorrhoea in the 6-month period before (February-July 2012) and after (February-July 2014) the introduction of self-taken extra-genital swabs. The rate of self-swabbing was determined in separate consecutive groups of 100 patients who had extragenital swabs in the same periods. 